\title{
A retrospective comparative exploratory study on two Methylentetrahydrofolate Reductase (MTHFR) polymorphisms in esophagogastric cancer: the A1298C MTHFR polymorphism is an independent prognostic factor only in neoadjuvantly treated gastric cancer patients
}

Susanne Blank ${ }^{1 \dagger}$, Sivaramakrishna Rachakonda ${ }^{2 \dagger}$, Gisela Keller ${ }^{3}$, Wilko Weichert ${ }^{4}$, Florian Lordick ${ }^{5}$, Rupert Langer ${ }^{6}$, Christoph Springfeld ${ }^{7}$, Thomas Bruckner ${ }^{8}$, Karen Becker ${ }^{3}$, Rajiv Kumar ${ }^{2+}$ and Katja Ott ${ }^{1 *+}$

\begin{abstract}
Background: Methylentetrahydrofolate reductase (MTHFR) plays a major role in folate metabolism and consequently could be an important factor for the efficacy of a treatment with 5-fluorouracil. Our aim was to evaluate the prognostic and predictive value of two well characterized constitutional MTHFR gene polymorphisms for primarily resected and neoadjuvantly treated esophagogastric adenocarcinomas.

Methods: 569 patients from two centers were analyzed (gastric cancer: 218, carcinoma of the esophagogastric junction (AEG II, III): 208 and esophagus (AEG I): 143). 369 patients received neoadjuvant chemotherapy followed by surgery, 200 patients were resected without preoperative treatment. The MTHFR C677T and A1298C polymorphisms were determined in DNA from peripheral blood lymphozytes. Associations with prognosis, response and clinicopathological factors were analyzed retrospectively within a prospective database (chi-square, log-rank, cox regression).
\end{abstract}

Results: Only the MTHFR A1298C polymorphisms had prognostic relevance in neoadjuvantly treated patients but it was not a predictor for response to neoadjuvant chemotherapy. The AC genotype of the MTHFR A1298C polymorphisms was significantly associated with worse outcome ( $p=0.02$, HR 1.47 (1.06-2.04). If neoadjuvantly treated patients were analyzed based on their tumor localization, the AC genotype of the MTHFR A1298C polymorphisms was a significant negative prognostic factor in patients with gastric cancer according to UICC $6^{\text {th }}$ edition (gastric cancer including AEG type II, III: HR 2.0, 95\% CI 1.3-2.0, $p=0.001$ ) and $7^{\text {th }}$ edition (gastric cancer without AEG II, III: HR 2.8, 95\% Cl 1.5-5.7, p=0.003), not for AEG I. For both definitions of gastric cancer the AC genotype was confirmed as an independent negative prognostic factor in cox regression analysis. In primarily resected patients neither the MTHFR A1298C nor the MTHFR C677T polymorphisms had prognostic impact.

\footnotetext{
* Correspondence: katja.ott@med.uni-heidelberg.de

${ }^{\dagger}$ Equal contributors

'Department of Surgery, University Hospital of Heidelberg, Im Neuenheimer

Feld 110, Heidelberg 69120, Germany

Full list of author information is available at the end of the article
} 
(Continued from previous page)

Conclusions: The MTHFR A1298C polymorphisms was an independent prognostic factor in patients with neoadjuvantly treated gastric adenocarcinomas (according to both UICC $6^{\text {th }}$ or $7^{\text {th }}$ definitions for gastric cancer) but not in AEG I nor in primarily resected patients, which confirms the impact of this enzyme on chemotherapy associated outcome.

Keywords: Esophagogastric adenocarcinoma, Prognostic factors, Folate metabolism, Methylentetrahydrofolate reductase, Genetic polymorphisms, C677T, A1298C

\section{Background}

Multimodal treatment is the standard of care for locally advanced adenocarcinomas of the esophagus or stomach since several randomized trials and meta-analyses have shown a prognostic benefit for (peri-) preoperative therapy versus surgery alone [1-4]. For patients who received multimodal treatment it is widely accepted that responding patients have a significantly better outcome than nonresponding patients [5-8]. However, depending on the therapy regimen applied, only $25-50 \%$ of patients respond to (peri-) preoperative treatment. Until now no molecular markers are available to predict response or survival in clinical routine and to tailor treatment individually. Despite the current guidelines favoring a multimodal treatment for locally advanced tumors, a relevant number of patients are still resected without preoperative treatment due to favorable tumor categories, differing local standards, individual risk factors or patients' choice.

Simple pretherapeutically available cliniocopathological factors like tumor localization, grading, content of signet ring cells and Laurén classification have been found to be associated with prognosis in patients with and without preoperative treatment in several studies [9-14]. So far, however these factors are not routinely used to tailor treatment or to stratify groups within clinical trials. Several studies on molecular and genetic prognostic and/or predictive markers in patients with gastric cancer have been published, but none of them (apart from HER-2 [15] in the palliative setting) gained clinical relevance $[16,17]$. Aside from tumor related factors, constitutional factors such as genetic polymorphisms are accepted to be associated with response and prognosis in adenocarcinomas of esophagus or stomach [18-21]. Most data exist for genetic polymorphisms being involved in pharmacodynamics and drug metabolism. Our own data on constitutional polymorphisms have shown to a large extent that the examined constitutional variants were associated rather with prognosis, than with response to preoperative treatment [22,23]. Only a few studies have investigated constitutional factors with the goal to clarify whether these factors are only relevant in the presence of chemotherapy or if they are prognostic factors irrespective of preoperative treatment [22].

Methylentetrahydrofolate reductase (MTHFR) plays a major role in the folate metabolism and consequently could be an important factor for the efficacy of a treatment with 5-fluorouracil (5-FU) [24-27].

The MTHFR single nucleotide polymorphism (SNP) C677T (rs 1801133) has shown to be associated with prognosis in gastric cancer patients in several studies $[23,28,29]$, however results are still conflicting [30] and the clinical relevance of this SNP has to be reproduced before clinical consequences can be drawn.

Another SNP in the MTHFR gene (A1298C) (rs 1801131) has also been investigated in several studies but results are less convincing than for C677T [31,32]. A recent study of our group found a significant association of this MTHFR gene A1298C polymorphism with prognosis in 258 neoadjuvantly treated esophagogastric adenocarcinomas [23].

In this study we focused on these two polymorphisms in the MTHFR gene to confirm the prognostic significance of the respective MTHFR gene polymorphisms in a larger group of esophagogastric cancer patients with neoadjuvant treatment as well as to test whether these polymorphisms can be used as a predictor for response to neoadjuvant chemotherapy. Additionally we wanted to test their relevance in a group of patients without perioperative chemotherapy.

\section{Methods}

569 patients with histologically proven adenocarcinoma of the esophagus (AEG I), esophagogastric junction (AEG II, III) or stomach (GC) were included in the study. Patients were treated in the Klinikum rechts der Isar, Munich from 1994-2005 ( $\mathrm{n}=361)$ or the University Hospital Heidelberg, Surgical Department from 2007-2010 ( $\mathrm{n}=235), 27$ patients were excluded due to non-operative treatment. 369 patients received neoadjuvant chemotherapy followed by surgery, while 200 patients were resected without preoperative treatment (Figure 1).

It is of note that for 244 of the 369 neoadjuvantly treated patients the results with shorter follow-up have been reported recently for both analyzed polymorphisms [23].

The study was approved by the ethical committee of the University of Heidelberg and of the Technical University of Munich and written informed consent was obtained from all patients. 


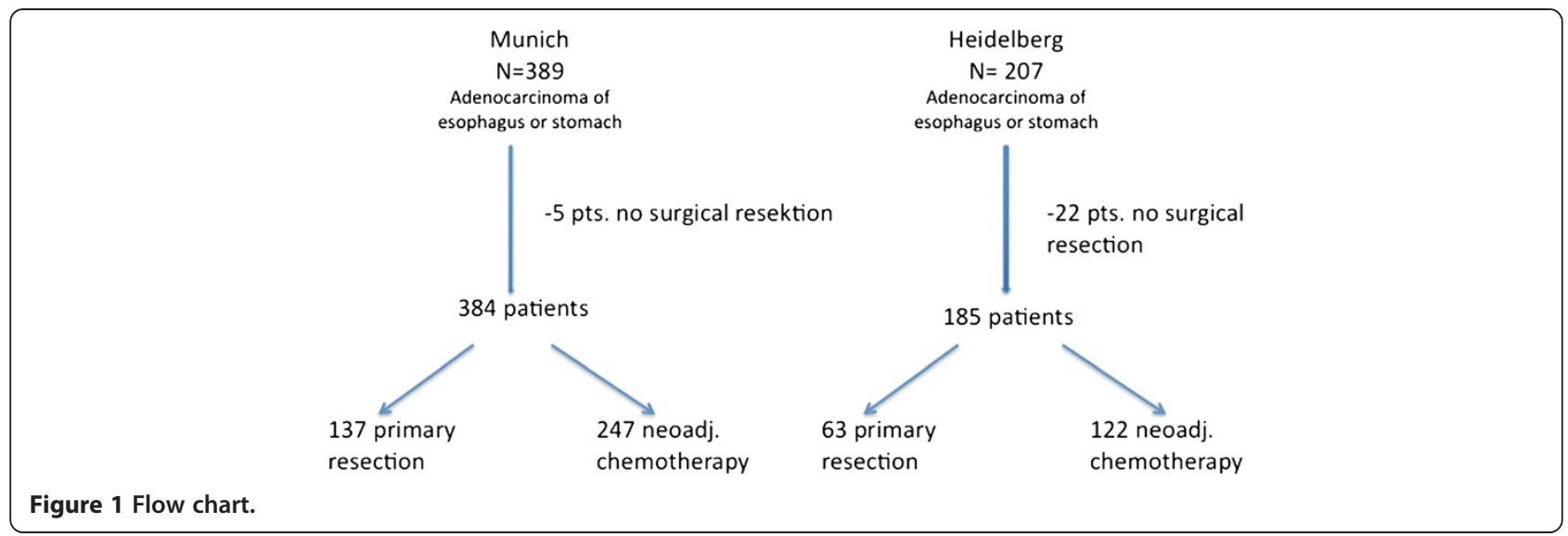

\section{Data assessment}

Demographic data, primary tumor localization, grading, type of resection, Lauren's subtype, ypTNM- and Rcategory, data on clinical and histopathological response as well as perioperative complications and mortality were documented prospectively in a database containing all patients with carcinoma of the esophagus or stomach in the two centers.

\section{Genotyping}

Blood was collected from patients before surgery. DNA from blood was isolated with Qiagen mini-preparation kits and genotyped for the two polymorphisms in the MTHFR gene (C677T and A1298C). Genotyping was performed using KASPar chemistry, a competitive allele-specific PCR genotyping system (http://www.lgcgenomics.com) according to manufacturer's instructions. The PCR was carried out in plates of 96 wells, in a total reaction volume of $8 \mu \mathrm{l}$ using $10 \mathrm{ng}$ of genomic DNA, $2 \mu \mathrm{l} 2 \mathrm{X}$ KASPar reaction mix and $0.11 \mu \mathrm{l}$ of the assay mix. The PCR conditions were: an initial denaturation at $94^{\circ} \mathrm{C}$ for 15 minutes, 10 touch-down cycles of $20 \mathrm{~s}$ at $94^{\circ} \mathrm{C}$ and $60 \mathrm{~s}$ at $61-55^{\circ} \mathrm{C}$ (temperature decrement by $0.6^{\circ} \mathrm{C}$ per cycle) and additional $25-30$ cycles at $20 \mathrm{~s}$ at $94^{\circ} \mathrm{C}$ and $60 \mathrm{~s}$ at $55^{\circ} \mathrm{C}$. Genotypes in amplified products were determined by differences in VIC and FAM fluorescent level in plate read operation on ABI PRISM 7900HT (Applied Biosystems, Foster City, CA) using SDS 2.2 Software. Post operation data were transferred to Microsoft Excel files and converted into genotype information. The genotype quality control was validated through DNA sequencing in $5 \%$ of the samples.

\section{Chemotherapy}

369 patients were treated with neoadjuvant chemotherapy: Munich $(n=247)$ : OLF/PLF regimen: either oxaliplatin $85 \mathrm{mg} / \mathrm{m}^{2}$ or cisplatin $50 \mathrm{mg} / \mathrm{m}^{2}$ on days $1,15,29$ and folinic acid $\left(500 \mathrm{mg} / \mathrm{m}^{2}\right.$ over $\left.2 \mathrm{~h}\right)$ plus fluorouracil $\left(2000 \mathrm{mg} / \mathrm{m}^{2}\right)$ on days $1,8,15,22,29$ and 36 , all repeated on day 49 , for patients with a good health status additionally paclitaxel $\left(80 \mathrm{mg} / \mathrm{m}^{2}\right)$ on days 0,14 and 28) was given [33]. Heidelberg $(n=122)$ : EOX-regimen: epirubicin $50 \mathrm{mg} / \mathrm{m} 2$ (day 1), oxaliplatin $130 \mathrm{mg} / \mathrm{m} 2$ (day 1 ), and capecitabin $1,250 \mathrm{mg} / \mathrm{m} 2$ (days 1-21), all repeated on day 22., PLF (see above), and FLOT: oxaliplatin $85 \mathrm{mg} / \mathrm{m} 2$ (day 1), docetaxel $50 \mathrm{mg} / \mathrm{m} 2$ (day 1), folinacid $200 \mathrm{mg} / \mathrm{m} 2$ (day 1), and 5-fluoruracil 2,600 mg/m2 (day 1 ), all repeated on day 15 [9].

\section{Surgery}

The type of surgery was performed according to the tumor localization and local standards: for patients with AEG I either an abominothoracic approach with intrathoracic anastomosis (Ivor Lewis procedure) [34] or a transhiatal esophagectomy [35], both including an abdominal D-2 lymphadenectomy, for patients with adenocarcinoma of the esophagogastric junction (AEG II, III) a transhiatal gastrectomy, in some cases an Ivor lewis procedure [36] or a transhiatal esophagectomy [35] was performed. Patients with gastric cancer received a total gastrectomy or subtotal gastrectomy [37] if an adequate proximal resection margin was possible. Both procedures included a D2-lymphadenectomy.

\section{Response to chemotherapy}

Clinical response was assessed after chemotherapy and before surgery comparing pre- and posttherapeutic computed tomography imaging and endoscopy. The evaluation was done by an interdisciplinary tumor board of the Klinikum rechts der Isar, Munich or by the Surgical Department of the University of Heidelberg. Criteria for response were a decrease of the maximal transversal tumor diameter of $>50 \%$ in CT and an estimated decrease in endoluminal tumor size of $>75 \%$ in endoscopy. Patients with minor response, no change or progressive disease were classified as nonresponder [38-40].

Histopathological response was assessed according to the Becker regression score [5,41]: tumor regression 
Table 1 Patients' characteristics and survival times according to clinicopathological factors

\begin{tabular}{|c|c|c|c|c|c|c|c|}
\hline \multicolumn{2}{|l|}{ Characteristics } & $\mathrm{n}$ & $\%$ & Median (months) & 3-Y-S (\%) & 5-Y-S (\%) & $p$ \\
\hline \multicolumn{8}{|l|}{ a: All patients } \\
\hline \multirow[t]{2}{*}{ Sex } & Male & 431 & 75.7 & 66.0 & 60.8 & 52.6 & \multirow[t]{2}{*}{0.637} \\
\hline & Female & 138 & 24.3 & 66.0 & 57.0 & 52.7 & \\
\hline Localization & AEG & 351 & 61.7 & 74.2 & 60.4 & 52.4 & \multirow[t]{2}{*}{0.81} \\
\hline UICC 7th edition & Gastric cancer & 212 & 37.3 & 62.2 & 58.7 & 52.3 & \\
\hline Localization & AEG I & 143 & 25.1 & 108.0 & 63.0 & 55.5 & 0.288 \\
\hline UICC 6 th edition & $A E G\|/\|+G C$ & 426 & 74.9 & 64.1 & 58.9 & 51.8 & \\
\hline \multirow[t]{2}{*}{ Laurén } & intestinal & 320 & 56.2 & 78.8 & 65.1 & 57.2 & \multirow[t]{2}{*}{0.029} \\
\hline & non-intestinal & 234 & 41.1 & 47.1 & 54.8 & 48.2 & \\
\hline \multirow[t]{2}{*}{ Grading } & low grade & 166 & 29.2 & 108.0 & 69.3 & 64.7 & \multirow[t]{2}{*}{0.012} \\
\hline & high grade & 395 & 69.4 & 54.1 & 56.4 & 47.8 & \\
\hline \multirow[t]{5}{*}{ pT } & pT0 & 38 & 6.7 & n.r. & 85.6 & 76.4 & \multirow[t]{5}{*}{$<0.001$} \\
\hline & pT1 & 52 & 9.1 & n.r. & 83.5 & 83.5 & \\
\hline & pT2 & 276 & 48.5 & 85.6 & 68.7 & 62.5 & \\
\hline & pT3 & 184 & 32.2 & 26.7 & 37.4 & 25.4 & \\
\hline & pT4 & 19 & 3.3 & 14.2 & 5.3 & 0.0 & \\
\hline \multirow[t]{5}{*}{$\mathrm{pN}$} & pNO & 242 & 42.5 & 101.9 & 76.8 & 69.6 & \multirow[t]{5}{*}{$<0.001$} \\
\hline & $\mathrm{pN} 1$ & 201 & 35.3 & 33.3 & 48.2 & 39.4 & \\
\hline & $\mathrm{pN} 2$ & 74 & 13 & 26.3 & 41.3 & 34.6 & \\
\hline & $\mathrm{pN3}$ & 28 & 4.9 & 18.9 & 25.4 & 25.4 & \\
\hline & $\mathrm{pNx}$ & 24 & 4.2 & n.r. & 78.5 & 78.5 & \\
\hline \multirow[t]{2}{*}{$\mathrm{pM}$} & $\mathrm{pMO}$ & 525 & 92.3 & 72.8 & 62.0 & 54.6 & \multirow[t]{2}{*}{0.001} \\
\hline & $\mathrm{pM1}$ & 44 & 7.7 & 23.6 & 36.3 & 32.2 & \\
\hline \multirow[t]{2}{*}{$\mathbf{R}$} & Ro & 476 & 83.7 & 74.2 & 64.7 & 56.7 & \multirow[t]{2}{*}{$<0.001$} \\
\hline & $\mathrm{R} 1 / \mathrm{R} 2$ & 93 & 16.3 & 20.3 & 34.2 & 32.2 & \\
\hline \multirow[t]{2}{*}{ Neoadjuvant chemotherapy } & yes & 369 & 64.9 & 74.2 & 61.5 & 52.6 & \multirow[t]{2}{*}{0.157} \\
\hline & no & 200 & 35.1 & 62.2 & 56.8 & 53.1 & \\
\hline Characteristics & & $\mathbf{n}$ & $\%$ & Median (months) & 3-Y-S (\%) & $5-Y-S(\%)$ & $\mathbf{p}$ \\
\hline \multicolumn{8}{|l|}{ b: Neoadjuvantly treated patients } \\
\hline \multirow[t]{2}{*}{ Sex } & Male & 309 & 83.7 & 78.8 & 63.2 & 53.7 & \multirow[t]{2}{*}{0.253} \\
\hline & Female & 60 & 16.3 & 48.6 & 53.0 & 46.9 & \\
\hline Localization & AEG & 287 & 77.8 & 78.8 & 63.4 & 54.4 & \multirow[t]{2}{*}{0.259} \\
\hline UICC 7th edition & Gastric cancer & 82 & 22.2 & 42.5 & 55.7 & 46.3 & \\
\hline Localization & AEG I & 139 & 37.7 & 108.0 & 62.8 & 55.3 & 0.653 \\
\hline UICC 6th edition & $A E G\|/\|+G C$ & 230 & 62.3 & 66.0 & 61.0 & 51.1 & \\
\hline Laurén & Intestinal & 220 & 59.6 & 108.0 & 67.3 & 58.7 & 0.014 \\
\hline & Non-intestinal & 140 & 37.9 & 40.5 & 54.4 & 44.6 & \\
\hline Grading & Low grade & 109 & 29.5 & 108.0 & 73.0 & 68.2 & 0.008 \\
\hline & High grade & 254 & 68.8 & 47.1 & 56.8 & 45.3 & \\
\hline pT & pT0 & 38 & 10.3 & n.r. & 85.6 & 76.4 & $<0.001$ \\
\hline & pT1 & 35 & 9.5 & n.r. & 83.2 & 83.2 & \\
\hline & pT2 & 169 & 45.8 & 101.9 & 68.7 & 60.3 & \\
\hline & pT3 & 116 & 31.4 & 26.9 & 39.5 & 25.2 & \\
\hline & pT4 & 11 & 3.0 & 14.2 & 0.0 & 0.0 & \\
\hline
\end{tabular}


Table 1 Patients' characteristics and survival times according to clinicopathological factors (Continued)

\begin{tabular}{|c|c|c|c|c|c|c|c|}
\hline \multirow[t]{5}{*}{$\overline{\mathrm{pN}}$} & $\mathrm{pNO}$ & 157 & 42.5 & 108.0 & 77.4 & 69.3 & $<0.001$ \\
\hline & $\mathrm{pN1}$ & 142 & 38.5 & 34.0 & 49.0 & 38.6 & \\
\hline & $\mathrm{pN} 2$ & 39 & 10.6 & 29.1 & 44.1 & 31.6 & \\
\hline & $\mathrm{pN3}$ & 17 & 4.6 & 18.9 & 38.5 & 38.5 & \\
\hline & $\mathrm{pNx}$ & 14 & 3.8 & n.r. & 77.9 & 77.9 & \\
\hline \multirow[t]{2}{*}{$\mathrm{pM}$} & $\mathrm{pMO}$ & 328 & 88.9 & 82.7 & 65.1 & 55.6 & $<0.001$ \\
\hline & pM1 & 41 & 11.1 & 23.6 & 33.4 & 28.6 & \\
\hline \multirow[t]{2}{*}{$\mathbf{R}$} & RO & 291 & 78.9 & n.r. & 69.0 & 58.4 & $<0.001$ \\
\hline & $\mathrm{R} 1 / \mathrm{R} 2$ & 78 & 21.1 & 20.3 & 32.7 & 30.4 & \\
\hline \multirow[t]{2}{*}{ Clinical response } & Responder & 105 & 28.5 & 39.2 & 82.6 & 74.2 & $<0.001$ \\
\hline & Nonresponder & 263 & 71.3 & 108.0 & 52.4 & 42.8 & \\
\hline \multirow[t]{2}{*}{ TRG } & $1 a, 1 b$ & 104 & 28.2 & n.r. & 83.8 & 77.4 & $<0.001$ \\
\hline & 2,3 & 263 & 71.3 & 39.2 & 52.5 & 42.1 & \\
\hline Characteristics & & $\mathbf{n}$ & $\%$ & Median (months) & 3-Y-S (\%) & 5-Y-S (\%) & $p$ \\
\hline \multicolumn{8}{|c|}{ c: Primarily resected patients } \\
\hline \multirow[t]{2}{*}{ Sex } & Male & 122 & 61.0 & 62.2 & 54.6 & 50.1 & 0.318 \\
\hline & Female & 78 & 39.0 & 66.0 & 60.5 & 57.5 & \\
\hline Localization & AEG & 64 & 32.0 & 32.6 & 46.9 & 44.3 & 0.049 \\
\hline UICC 7th edition & Gastric cancer & 131 & 65.5 & 64.1 & 60.7 & 56.4 & \\
\hline Localization & AEG I & 4 & 2.0 & n.r. & 75.0 & 75.0 & 0.619 \\
\hline UICC 6th edition & AEG $\|/\|+\mathrm{GC}$ & 196 & 98.0 & 62.2 & 56.5 & 52.9 & \\
\hline \multirow[t]{2}{*}{ Laurén } & Intestinal & 100 & 50.0 & 62.2 & 59.8 & 53.4 & 0.807 \\
\hline & Non-intestinal & 94 & 47.0 & 64.1 & 55.7 & 54.0 & \\
\hline \multirow[t]{2}{*}{ Grading } & Low grade & 57 & 28.5 & 62.2 & 61.5 & 55.9 & 0.526 \\
\hline & High grade & 141 & 70.5 & 64.1 & 55.8 & 52.2 & \\
\hline \multirow[t]{5}{*}{ pT } & pTO & 0 & 0.0 & & & & $<0.001$ \\
\hline & pT1 & 17 & 8.5 & n.r. & 86.5 & 86.5 & \\
\hline & pT2 & 107 & 53.5 & 85.6 & 68.5 & 65.4 & \\
\hline & pT3 & 68 & 34.0 & 25.8 & 33.2 & 26.6 & \\
\hline & pT4 & 8 & 4.0 & 10.3 & 12.5 & 12.5 & \\
\hline \multirow[t]{5}{*}{$\mathrm{pN}$} & pNO & 85 & 42.5 & 85.6 & 76.0 & 70.2 & $<0.001$ \\
\hline & $\mathrm{pN1}$ & 59 & 29.5 & 32.0 & 45.4 & 41.6 & \\
\hline & $\mathrm{pN} 2$ & 35 & 17.5 & 21.7 & 37.9 & 28.4 & \\
\hline & $\mathrm{pN3}$ & 11 & 5.5 & 16.1 & 9.1 & 9.1 & \\
\hline & $\mathrm{pNx}$ & 10 & 5.0 & n.r. & 78.8 & 78.8 & \\
\hline \multirow[t]{2}{*}{$\mathrm{pM}$} & $\mathrm{pMO}$ & 197 & 98.5 & 62.2 & 56.6 & 52.9 & 0.684 \\
\hline & pM1 & 3 & 1.5 & n.r. & 66.7 & 66.7 & \\
\hline \multirow[t]{2}{*}{ R } & Ro & 185 & 92.5 & 64.1 & 57.8 & 53.9 & 0.144 \\
\hline & $\mathrm{R} 1 / \mathrm{R} 2$ & 15 & 7.5 & 18.1 & 44.4 & 0.0 & \\
\hline
\end{tabular}

MS = median survival, 3-Y-S=3-Year-Survival, 5-Y-S = 5-Year-Survival, AEG = adenocarcinoma of the esophagogastric junction, TRG = tumor regression grade, n.r. $=$ not reached.

grade (TRG) 1a (complete regression) and $1 \mathrm{~b}(<10 \%$ residual tumor) were classified as histopathological response, TRG 2 (10-50\% residual tumor) and 3 (>50\% residual tumor) as nonresponse.

\section{Follow-up}

Follow-up was done according to the local guidelines. Patients who were not followed in one of the two centers were contacted by phone to obtain follow-up data. 
Table 2 Genotype frequencies: MTHFR C677T

\begin{tabular}{|c|c|c|c|c|c|}
\hline & & \multicolumn{2}{|c|}{ MTHFR C677T } & \multirow[b]{2}{*}{$\pi$} & \multirow[t]{2}{*}{$p$} \\
\hline & & CC & CT & & \\
\hline All patients & & $254(44.6 \%)$ & $262(46.0 \%)$ & $53(9.3 \%)$ & \\
\hline \multirow[t]{2}{*}{ Status } & Alive & $143(42.2 \%)$ & $162(47.8 \%)$ & $34(10 \%)$ & 0.342 \\
\hline & Dead & 111 (48.3\%) & $100(43.5 \%)$ & $19(8.3 \%)$ & \\
\hline \multirow[t]{2}{*}{ Sex } & Male & 189 (43.9\%) & $202(46.9 \%)$ & $40(9.3 \%)$ & 0.775 \\
\hline & Female & $65(47.1 \%)$ & $60(43.5 \%)$ & $13(9.4 \%)$ & \\
\hline Localization & AEG & $153(43.6 \%)$ & $169(48.1 \%)$ & $29(8.3 \%)$ & 0.337 \\
\hline UICC 7th edtiion & Gastric cancer & 99 (46.7\%) & $90(42.5 \%)$ & $23(10.8 \%)$ & \\
\hline Localization & AEG I & $63(44.1 \%)$ & $68(47.6 \%)$ & $12(8.4 \%)$ & 0.868 \\
\hline UICC 6th edition & AEG $\|/\|+\mathrm{GC}$ & $191(44.8 \%)$ & $194(45.5 \%)$ & $41(9.6 \%)$ & \\
\hline \multirow[t]{2}{*}{ Laurén } & Intestinal & $137(42.8 \%)$ & $151(47.2 \%)$ & $32(10.0 \%)$ & 0.731 \\
\hline & Non-intestinal & 107 (45.7\%) & 107 (45.7\%) & $20(8.5 \%)$ & \\
\hline \multirow[t]{2}{*}{ Grading } & Low grade & $74(44.6 \%)$ & 75 (45.2\%) & 17 (10.2\%) & 0.89 \\
\hline & High grade & $174(44.1 \%)$ & 185 (46.8\%) & $36(9.1 \%)$ & \\
\hline \multirow[t]{5}{*}{ pT } & pTO & $14(36.8 \%)$ & $21(55.3 \%)$ & $3(7.9 \%)$ & 0.751 \\
\hline & pT1 & $20(38.5 \%)$ & $28(53.8 \%)$ & $4(7.7 \%)$ & \\
\hline & pT2 & $134(48.6 \%)$ & $118(42.8 \%)$ & $24(8.7 \%)$ & \\
\hline & pT3 & $78(42.4 \%)$ & $86(46.7 \%)$ & $20(10.9 \%)$ & \\
\hline & pT4 & $8(42.1 \%)$ & $9(47.4 \%)$ & $2(10.5 \%)$ & \\
\hline \multirow[t]{5}{*}{$\mathrm{pN}$} & $\mathrm{pNO}$ & $109(45.0 \%)$ & $115(47.5 \%)$ & $18(7.4 \%)$ & 0.642 \\
\hline & $\mathrm{pN} 1$ & $93(46.3 \%)$ & $88(43.8 \%)$ & $20(10.0 \%)$ & \\
\hline & $\mathrm{pN} 2$ & $31(41.9 \%)$ & $33(44.6 \%)$ & $10(13.5 \%)$ & \\
\hline & $\mathrm{pN3}$ & $12(42.9 \%)$ & $15(53.6 \%)$ & $1(3.6 \%)$ & \\
\hline & $\mathrm{pNx}$ & $9(37.5 \%)$ & $11(45.8 \%)$ & $4(16.7 \%)$ & \\
\hline \multirow[t]{2}{*}{$\mathrm{pM}$} & $\mathrm{pM0}$ & $236(45.0 \%)$ & $243(46.3 \%)$ & $46(8.8 \%)$ & 0.292 \\
\hline & pM1 & $18(40.9 \%)$ & $19(43.2 \%)$ & $7(15.9 \%)$ & \\
\hline \multirow[t]{2}{*}{$\mathbf{R}$} & RO & $213(44.7 \%)$ & $218(45.8 \%)$ & $45(9.5 \%)$ & 0.948 \\
\hline & $\mathrm{R} 1 / \mathrm{R} 2$ & $41(44.1 \%)$ & $44(47.3 \%)$ & $8(8.6 \%)$ & \\
\hline \multirow[t]{2}{*}{ Neoadjuvant chemotherapy } & yes & $161(43.6 \%)$ & $179(48.5 \%)$ & $29(7.9 \%)$ & 0.135 \\
\hline & no & $93(46.5 \%)$ & $83(41.5 \%)$ & $24(12.0 \%)$ & \\
\hline \multirow[t]{2}{*}{ Clinical response } & Responder & $44(41.9 \%)$ & $52(49.5 \%)$ & $9(8.6 \%)$ & 0.885 \\
\hline & Nonresponder & $117(44.5 \%)$ & $126(47.9 \%)$ & $20(7.6 \%)$ & \\
\hline \multirow[t]{2}{*}{ TRG } & $1 \mathrm{a}, 1 \mathrm{~b}$ & $44(42.3 \%)$ & $53(51.0 \%)$ & $7(6.7 \%)$ & 0.783 \\
\hline & 2,3 & $116(44.1 \%)$ & $125(47.5 \%)$ & $22(8.4 \%)$ & \\
\hline
\end{tabular}

$\mathrm{AEG}=$ adenocarcinoma of the esophagogastric junction, $\mathrm{GC}=$ gastric cancer, $\mathrm{TRG}=$ tumor regression grade.

Median time of follow-up of the surviving patients was $41.1+/-25.8$ months.

\section{Statistical analysis}

For statistical analysis we used SPSS 20.0 (IBM Inc. Chicago). Quantitative data is presented as mean +/standard deviation. Survival curves were estimated by the Kaplan-Meier method and presented in months from time of diagnosis to death. Differences in survival times were calculated using the log-rank test. For univariate and multivariate analysis we used the Cox proportional hazard model. For correlation between different parameters we used the Chi-square-test (two-sided) where appropriate. P-values $<0.05$ were considered as statistically significant.

\section{Results}

230 patients out of 569 have died. Median survival of the entire population was 66.0 months. Median survival of patients treated in Munich was 72.8 months, in Heidelberg 85.6 months. 
Table 3 Genotype frequencies: MTHFR A1298C

\begin{tabular}{|c|c|c|c|c|c|}
\hline & & \multicolumn{2}{|c|}{ MTHFR A1298C } & \multirow[b]{2}{*}{$\mathrm{CC}$} & \multirow[t]{2}{*}{$p$} \\
\hline & & AA & $A C$ & & \\
\hline All patients & & $244(42.9 \%)$ & $268(47.1 \%)$ & $54(9.5 \%)$ & \\
\hline \multirow[t]{2}{*}{ Status } & Alive & $152(45.1 \%)$ & $149(44.2 \%)$ & $36(10.7 \%)$ & 0.162 \\
\hline & Dead & $92(40.2 \%)$ & 119 (52.0\%) & $18(7.9 \%)$ & \\
\hline \multirow[t]{2}{*}{ Sex } & Male & $184(43.0 \%)$ & 204 (47.1\%) & $40(9.3 \%)$ & 0.945 \\
\hline & Female & $60(43.5 \%)$ & $64(46.4 \%)$ & $14(10.1 \%)$ & \\
\hline Localization & AEG & $152(43.7 \%)$ & $164(47.1 \%)$ & $32(9.2 \%)$ & 0.793 \\
\hline UICC 7th edtition & Gastric cancer & 87 (41.0\%) & $103(48.6 \%)$ & $22(10.4 \%)$ & \\
\hline Localization & AEG I & $60(42.6 \%)$ & $68(48.2 \%)$ & $13(9.2 \%)$ & 0.968 \\
\hline UICC 6th edition & AEG $\|/\|+\mathrm{GC}$ & $184(43.3 \%)$ & $200(47.1 \%)$ & $41(9.6 \%)$ & \\
\hline \multirow[t]{2}{*}{ Laurén } & Intestinal & $140(44.0 \%)$ & $150(47.2 \%)$ & $28(8.8 \%)$ & 0.835 \\
\hline & Non-intestinal & $100(42.9 \%)$ & 109 (46.8\%) & $24(10.3 \%)$ & \\
\hline \multirow[t]{2}{*}{ Grading } & Low grade & 70 (42.4\%) & 77 (46.7\%) & 18 (10.9\%) & 0.76 \\
\hline & High grade & $172(43.8 \%)$ & $186(47.3 \%)$ & $35(8.9 \%)$ & \\
\hline \multirow[t]{5}{*}{ pT } & pTO & $16(42.1 \%)$ & $18(47.4 \%)$ & $4(10.5 \%)$ & 0.701 \\
\hline & pT1 & 17 (33.3\%) & $28(54.9 \%)$ & $6(11.8 \%)$ & \\
\hline & pT2 & 119 (43.3\%) & $125(45.5 \%)$ & $31(11.3 \%)$ & \\
\hline & pT3 & $83(45.4 \%)$ & $88(48.1 \%)$ & $12(6.6 \%)$ & \\
\hline & pT4 & $9(47.4 \%)$ & $9(47.4 \%)$ & $1(5.3 \%)$ & \\
\hline \multirow[t]{5}{*}{$\mathrm{pN}$} & $\mathrm{pNO}$ & $98(40.7 \%)$ & $118(49.0 \%)$ & $25(10.4 \%)$ & 0.654 \\
\hline & $\mathrm{pN} 1$ & $97(48.5 \%)$ & $84(42.0 \%)$ & $19(9.5 \%)$ & \\
\hline & $\mathrm{pN} 2$ & $30(41.1 \%)$ & $37(50.7 \%)$ & $6(8.2 \%)$ & \\
\hline & $\mathrm{pN3}$ & $9(32.1 \%)$ & $16(57.1 \%)$ & $3(10.7 \%)$ & \\
\hline & $\mathrm{pNx}$ & $10(41.7 \%)$ & $13(54.2 \%)$ & $1(4.2 \%)$ & \\
\hline \multirow[t]{2}{*}{$\mathrm{pM}$} & $\mathrm{pM0}$ & $226(43.3 \%)$ & $244(46.7 \%)$ & $52(10.0 \%)$ & 0.402 \\
\hline & pM1 & $18(40.9 \%)$ & $24(54.5 \%)$ & $2(4.5 \%)$ & \\
\hline \multirow[t]{2}{*}{$\mathbf{R}$} & RO & $199(42.0 \%)$ & $226(47.7 \%)$ & $49(10.3 \%)$ & 0.239 \\
\hline & $\mathrm{R} 1 / \mathrm{R} 2$ & $45(48.9 \%)$ & $42(45.7 \%)$ & $5(5.4 \%)$ & \\
\hline \multirow[t]{2}{*}{ Neoadjuvant chemotherapy } & Yes & $156(42.6 \%)$ & $179(48.9 \%)$ & $31(8.5 \%)$ & 0.4 \\
\hline & No & $88(44.0 \%)$ & 89 (44.5\%) & $23(11.5 \%)$ & \\
\hline \multirow[t]{2}{*}{ Clinical response } & Responder & $46(43.8 \%)$ & $49(46.7 \%)$ & $10(9.5 \%)$ & 0.812 \\
\hline & Nonresponder & $109(41.9 \%)$ & $130(50.0 \%)$ & $21(8.1 \%)$ & \\
\hline \multirow[t]{2}{*}{ TRG } & $1 \mathrm{a}, 1 \mathrm{~b}$ & $41(39.4 \%)$ & $55(52.9 \%)$ & $8(7.7 \%)$ & 0.628 \\
\hline & 2,3 & $114(43.8 \%)$ & $123(47.3 \%)$ & $23(8.8 \%)$ & \\
\hline
\end{tabular}

$\mathrm{AEG}=$ adenocarcinoma of the esophagogastric junction, $\mathrm{GC}=$ gastric cancer, $\mathrm{TRG}=$ tumor regression grade.

Patients' characteristics including survival times are presented in Table 1.

Relevant prognostic factors were Laurén's type $(\mathrm{p}=0.029)$, Grading ( $\mathrm{p}=0.012),(\mathrm{y}) \mathrm{pT}$-category $(\mathrm{p}<0.0000001),(\mathrm{y})$ pN-category $(\mathrm{p}<0.0000001),(y)$ pM-category $(\mathrm{p}=0.001)$, R-category $(\mathrm{p}<0.0000001)$, additionally clinical response $(\mathrm{p}<0.0000001)$ and histopathological response $(\mathrm{p}<0.0000001)$ for patients treated with neoadjuvant chemotherapy.

\section{Genotype frequencies and correlation with clinicopathological factors}

The genotype frequencies were in accordance with the Hardy-Weinberg-equilibrium. Frequencies of the individual genotypes are presented in Tables 2 and 3. No individual genotype showed any statistical correlation with a clinicopathological factor listed above, also if analyzed separately for the respective tumor entities (AEG versus 


\begin{tabular}{|c|c|c|c|c|c|}
\hline & & $\mathrm{n}$ & MS (months) & $\mathrm{p}$ & HR $(95 \% \mathrm{Cl})$ \\
\hline \multicolumn{6}{|l|}{ a: All patients } \\
\hline \multirow[t]{5}{*}{ MTHFR C677T } & $\mathrm{CC}$ & 254 & 54.1 & 0.412 & \\
\hline & $\mathrm{CT}$ & 262 & 82.7 & & $0.88(0.67-1.15)$ \\
\hline & $\pi$ & 53 & n.r. & & $0.75(0.46-1.22)$ \\
\hline & $\mathrm{CC}$ & 254 & 54.1 & 0.237 & \\
\hline & $C T+\Pi$ & 315 & 82.7 & & $0.86(0.66-1.11)$ \\
\hline \multirow[t]{6}{*}{ MTHFR A1298C } & AA & 244 & 82.7 & 0.036 & \\
\hline & $A C$ & 268 & 47.1 & & $1.31(1.0-1.72)$ \\
\hline & $\mathrm{CC}$ & 54 & 74.2 & & $0.77(0.47-1.28)$ \\
\hline & $A C$ & 268 & 47.1 & 0.016 & \\
\hline & $A A+C C$ & 301 & 82.7 & & $0.73(0.56-0.94)$ \\
\hline & & $\mathrm{n}$ & MS (months) & $\mathrm{p}$ & HR $(95 \% \mathrm{Cl})$ \\
\hline \multicolumn{6}{|c|}{ b: Neoadjuvantly treated patients } \\
\hline \multirow[t]{5}{*}{ MTHFR C677T } & $\mathrm{CC}$ & 161 & 55.4 & 0.745 & \\
\hline & $\mathrm{CT}$ & 179 & 82.7 & & $0.98(0.7-1.38)$ \\
\hline & $\pi$ & 29 & n.r. & & $0.77(0.4-1.51)$ \\
\hline & CC & 161 & 55.4 & 0.77 & \\
\hline & $C T+\Pi$ & 208 & 82.7 & & $0.95(0.69-1.32)$ \\
\hline \multirow[t]{6}{*}{ MTHFR A1298C } & AA & 156 & 101.9 & 0.063 & \\
\hline & $A C$ & 179 & 47.1 & & $1.43(1.02-2.01)$ \\
\hline & $\mathrm{CC}$ & 31 & 74.2 & & $0.85(0.44-1.67)$ \\
\hline & $A C$ & 179 & 47.1 & 0.02 & \\
\hline & $\mathrm{AA}+\mathrm{CC}$ & 190 & 101.9 & & $0.68(0.49-0.94)$ \\
\hline & & $\mathbf{n}$ & MS (months) & $\mathrm{p}$ & HR $(95 \% \mathrm{Cl})$ \\
\hline \multicolumn{6}{|c|}{ c: Primarily resected patients } \\
\hline \multirow[t]{5}{*}{ MTHFR C677T } & CC & 93 & 54.1 & 0.295 & \\
\hline & $\mathrm{CT}$ & 83 & n.r. & & $0.72(0.45-1.15)$ \\
\hline & $\pi$ & 24 & 66.0 & & $0.69(0.34-1.4)$ \\
\hline & $\mathrm{CC}$ & 93 & 54.1 & 0.119 & \\
\hline & $C T+T$ & 107 & 66.0 & & \\
\hline \multirow[t]{5}{*}{ MTHFR A1298C } & AA & 88 & 66.0 & 0.366 & \\
\hline & $A C$ & 89 & 47.6 & & $0.16(0.74-1.82)$ \\
\hline & $\mathrm{CC}$ & 23 & 62.2 & & $0.68(0.31-1.46)$ \\
\hline & $A C$ & 89 & 47.6 & 0.295 & \\
\hline & $A A+C C$ & 111 & 66.0 & & $0.79(0.52-1.22)$ \\
\hline
\end{tabular}

GC) and treatment groups (primary resection versus neoadjuvant treatment).

\section{Genotypes and survival}

\section{Survival and type of treatment}

Survival analysis was conducted for the whole study cohort as well as for neoadjuvantly and primarily resected patients separately. The MTHFR C677T polymorphisms did not show a statistically significant influence on survival times in all patients $(\mathrm{p}=0.412)$ nor in any analyzed subgroup (in neoadjuvantly treated patients $\mathrm{p}=$ 0.745 , in primarily resected patients $\mathrm{p}=0.295$ ), although there was a slight trend for shorter survival of patients with the CC genotype (54.1 months median in patients with CC genotype versus 82.7 months in patients with CT and TT genotype, $\mathrm{p}=0.237$ ), Table $4 \mathrm{a}$.

In contrast, the MTHFR A1298C polymorphisms were prognostically relevant $(\mathrm{p}=0.036)$ in all patients. The 


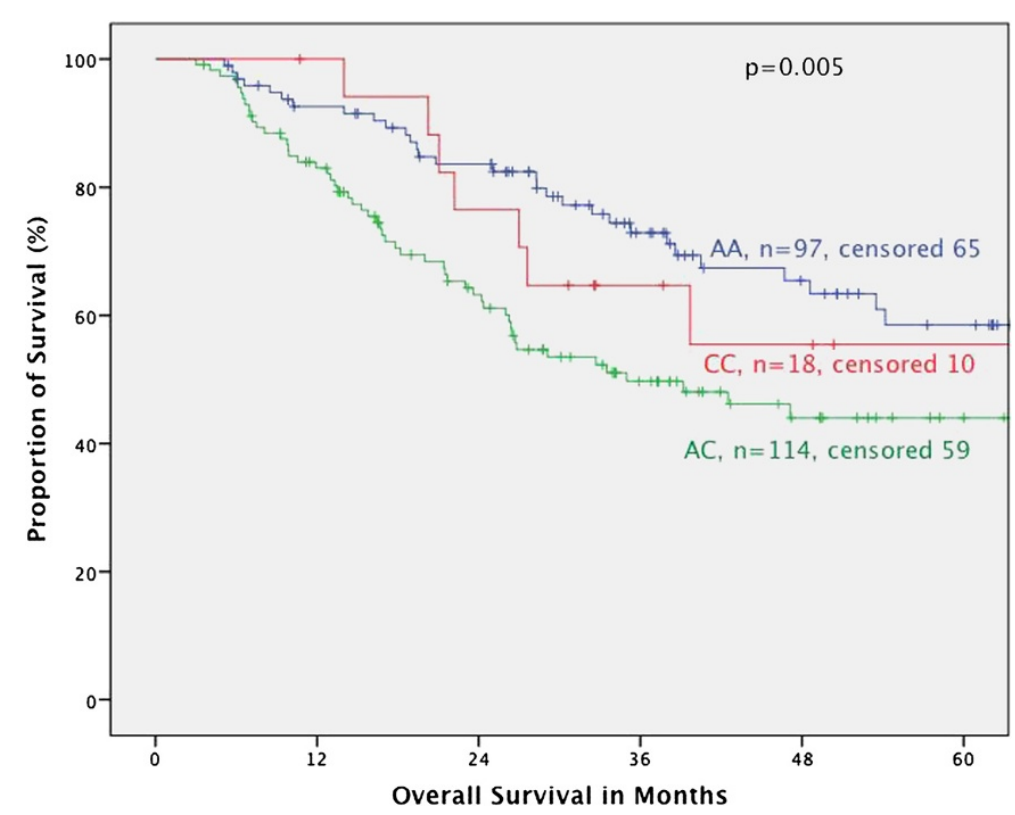

Figure 2 Survival in gastric cancer patients according to UICC 6th edition, MTHFR A1298C. Legend: Subgroup analysis for patients with adenocarcinoma of the esophagogastric junction type II, III and stomach dependent on polymorphisms of MTHFR A1298C. Median Survival times: AA 101.9 months, CC 74.2 months, AC 35.0 months, $p=0.005$.

AC genotype was associated with a worse outcome compared to AA and CC. As we did not find a prognostic difference between the AA and CC genotype, the two homozygous genotypes were combined and analyzed together: 47.1 months median for AC genotype versus 82.7 months for AA/CC genotype, $\mathrm{p}=0.016$, see Table $4 \mathrm{a}$.
The prognostic effect of MTHFR A1298C might be caused by the neoadjuvant treatment as it could not be shown in primarily resected patients.

The survival of the neoadjuvantly treated and primarily resected patients are presented in detail in Table $4 \mathrm{~b}$ and $\mathrm{c}$. In neoadjvuantly treated patients the

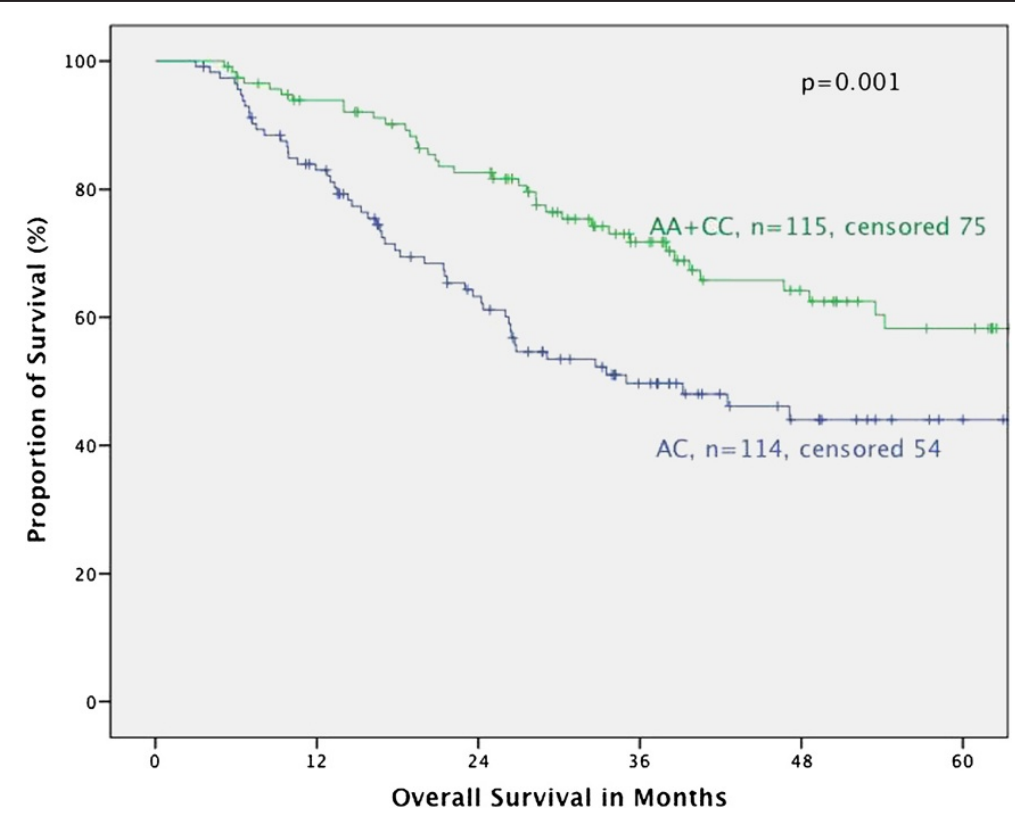

Figure 3 Survival in gastric cancer patients according to UICC 6th edition, MTHFR A1298C AC versus AA/CC. Legend: Subgroup analysis for patients with adenocarcinoma of the esophagogastric junction type II, III and stomach dependent on polymorphisms of MTHFR A1298C, comparing AC genoytpe to AA and CC genotype. Median Survival times: AA/CC 82.7 months, AC 35.0 months, $p=0.001$ ). 


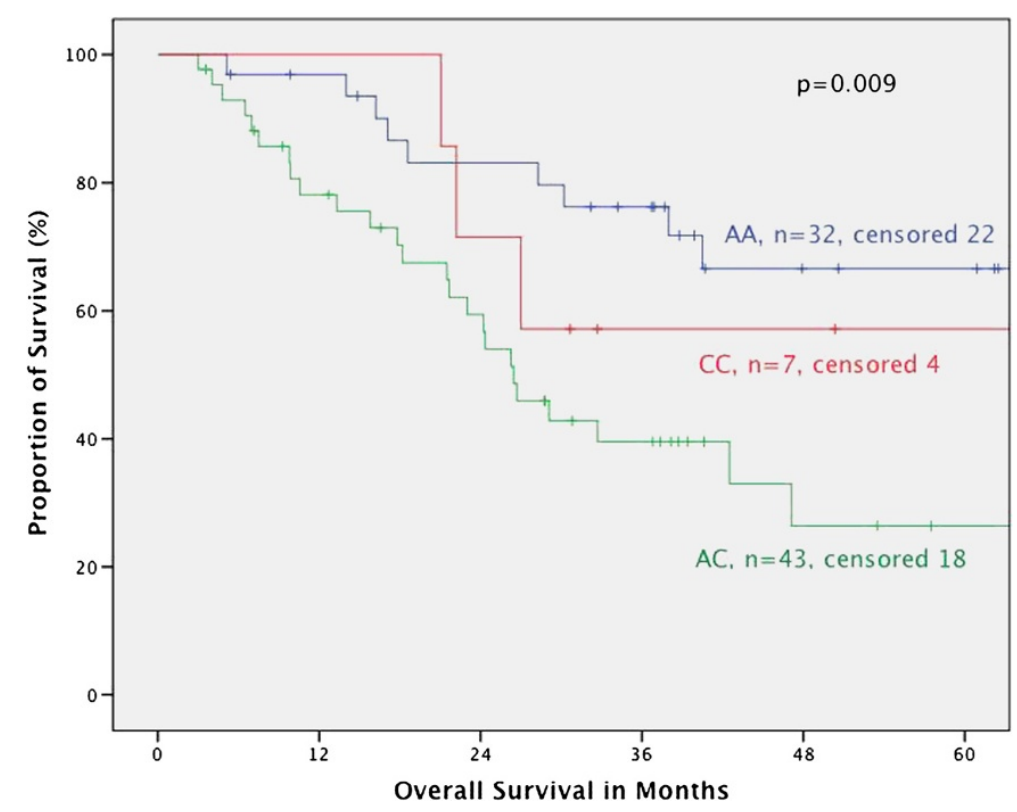

Figure 4 Survival in gastric cancer patients according to UICC 7th edition, MTHFR A1298C. Legend: Subgroup analysis for patients with adenocarcinoma of the stomach dependent on polymorphisms of MTHFR A1298C. Median Survival times: AA 82.7 months, CC not reached, AC 26.5 months, $p=0.009$.

A1298C polymorphisms showed a survival benefit for the $\mathrm{AA}$ and $\mathrm{CC}$ genotypes, compared to the $\mathrm{AC}$ genotype $(\mathrm{p}=0.02)$. In primarily resected patients the polymorphisms of both gene loci were not different in survival.

\section{Survival in respect of tumor localization}

Furthermore, the association of the respective genotypes with prognosis was tested stratified for the different tumor localizations. In primarily resected patients we found no correlation between MTHFR polymorphisms

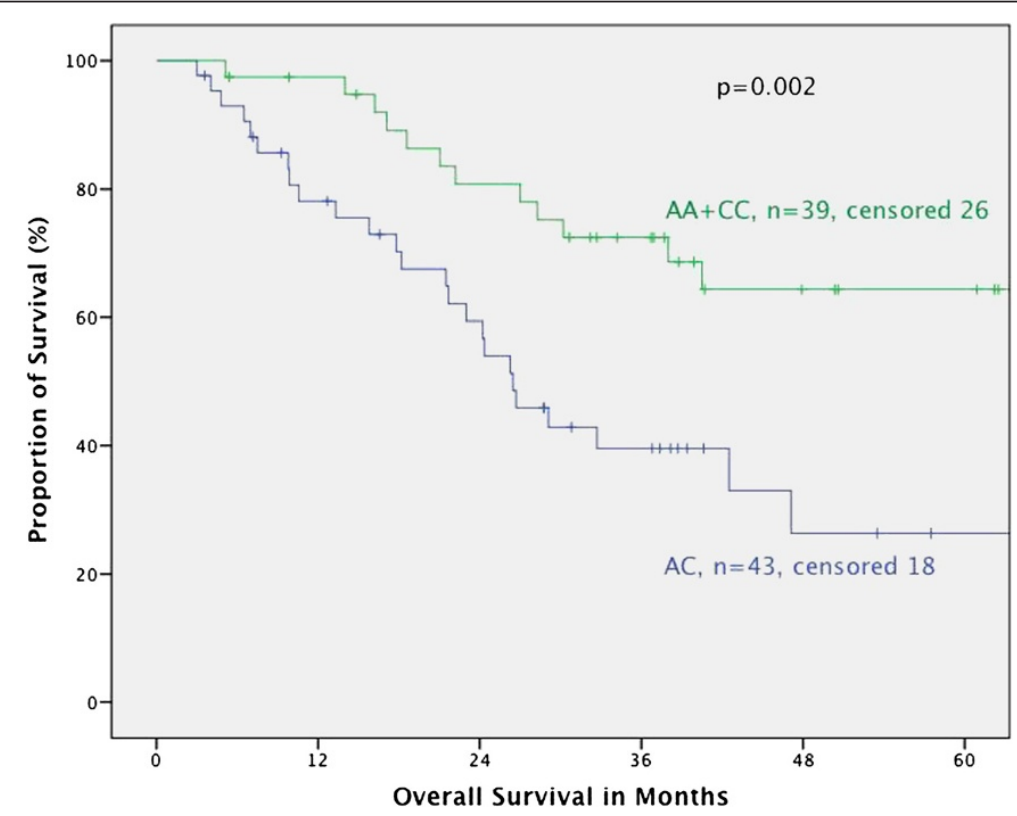

Figure 5 Survival in gastric cancer patients according to UICC 7th edition, MTHFR A1298C AC versus AA/CC. Legend: Subgroup analysis for patients with adenocarcinoma of the stomach dependent on polymorphisms of MTHFR A1298C, comparing AC genoytpe to AA and CC genotype. Median Survival times: AA/CC not reached, AC 26.5 months, $p=0.002$. 
Table 5 Multivariate analysis in neoadjuvantly treated patients with gastric cancer including AEG II, III (UICC 6th)

\begin{tabular}{lllll}
\hline & & HR & $\mathbf{9 5 \%} \mathbf{C l}$ & $\mathbf{p}$ \\
\hline $\mathbf{p T}$ & pT0 & 1.00 & & 0.002 \\
& pT1 & 2.16 & $0.2-20.8$ & \\
& pT2 & 5.10 & $0.7-37.5$ & \\
& pT3 & 9.80 & $1.3-74.3$ & \\
& pT4 & 14.50 & $1.7-122.9$ & \\
$\mathbf{R}$ & R0 & 1.00 & & 0.001 \\
& R1/2 & 2.20 & $1.3-3.5$ & \\
Clinical response & yes & 1.00 & & 0.026 \\
& no & 2.10 & $1.1-4.2$ & \\
MTHFR A1298C & AA & 1.00 & & 0.01 \\
& AC & 2.00 & $1.3-3.2$ & \\
& CC & 1.49 & $0.7-3.3$ & \\
\hline
\end{tabular}

and prognosis for the different localizations. In neoadjuvantly treated patients both polymorphisms had no prognostic impact in adenocarcinomas of the esophagus (AEG I) nor if taken all junctional tumors (AEG I, II, III) together according to the UICC $7^{\text {th }}$ classification. This is in contrast to patients with adenocarcinoma of the stomach: according to the old classification (gastric cancer including AEG II, III, UICC $6^{\text {th }}$ ) (Figures 2 and 3 ) as well as in gastric cancer according to the new classification (gastric cancer without AEG II, III, UICC $7^{\text {th }}$ ) the MTHFR A1298C polymorphism was a prognostic factor ( $p=0.005$ and 0.009 respectively), (Figures 4 and 5). The AC genotype had a significantly worse prognosis compared to the rest in gastric cancer defined by the $\mathrm{UICC}^{\text {th }}(\mathrm{p}=0.001$, HR $2.0(1.3-3.0))$ and the UICC $7^{\text {th }}$ $(\mathrm{p}=0.003$, HR $2.8(1.5-5.7))$.

\section{Multivariate analysis}

In neoadjuvantly treated gastric cancer patients according to UICC $6^{\text {th }}$ edition multivariate analysis (forward proportional hazard model) (including the univariate

Table 6 Multivariate Analysis in neoadjuvantly treated patients with gastric cancer without AEG II, III (UICC 7th)

\begin{tabular}{lllll}
\hline & & HR & $\mathbf{9 5 \%} \mathbf{C l}$ & $\mathbf{p}$ \\
\hline $\mathbf{R}$ & R0 & 1.00 & & 0.001 \\
& R1/2 & 3.40 & $1.7-6.8$ & \\
Clinical response & yes & 1.00 & & 0.021 \\
& no & 10.30 & $1.4-75.5$ & \\
MTHFR A1298C & AA & 1.00 & & 0.028 \\
& AC & 2.80 & $1.3-5.9$ & \\
& CC & 2.00 & $0.5-7.6$ & \\
\hline
\end{tabular}

significant factors grading, pT-, pN-category, R-category, clinical and histopathological response, MTHFR A1298C polymorphisms as well as gender and age for adjustment) revealed pT-category $(\mathrm{p}=0.002), \quad \mathrm{R}$-category $(p=0.001)$, clinical response $(p=0.026)$ and MTHFR A1298C $(\mathrm{p}=0.01)$ as independent prognostic factors. In gastric cancer patients according to UICC $7^{\text {th }}$ edition (including grading, pT-category, R-category, clinical response and MTHFR A1298C polymorphisms as well as gender and age) R-category ( $\mathrm{p}=0.001)$, clinical response $(\mathrm{p}=0.021)$ and MTHFR A1298C $(\mathrm{p}=0.028)$ were identified as independent prognostic factors (Tables 5 and 6). All prognostic factors were confirmed by the backward proportional hazard model.

\section{Discussion}

Our study revealed the AC genotype of the MTHFR A1298C as a predictor of poor prognosis in patients with gastric cancer. However, this genotype was only a prognostic marker after neoadjuvant treatment not in primarily resected patients. This gives a clear hint towards the contribution of the chemotherapy on the prognostic impact of this polymorphism. Additionally the prognostic influence seems to be limited to gastric cancer (UICC $6^{\text {th }}$ or $7^{\text {th }}$ edition) since it was not apparent for adenocarcinomas of the esophagus.

Both examined MTHFR polymorphisms are known to be functionally relevant. The variants (CT and TT) of MTHFR C677T polymorphisms are associated with decreased activity of MTHFR, which results in higher homocystein levels and lower plasma folate levels [42,43]. Similarly, the A1298C variants (AC and CC) are associated with a lower enzyme activity, but results in literature are less final and conclusive than for C677T [31,32,44]. Our findings on MTHFR C677T are not statistically significant, but the trend for longer survival of the variants of C677T (CT and TT) would be in line with the functional hypothesis.

Furthermore MTHFR is thought to play an important role in response to fluoropyrimidine containing chemotherapy. A decreased activity of MTHFR results in higher 5,10-methylene tetrahydrofolate levels which leads to inhibition of thimidylate synthase and consequently to DNA damage [45-47] leading theoretically to an increased response and survival. This simplified theoretical approach cannot be confirmed by our data. Our results are in part conflicting, which points out the complexity of chemotherapy response and prognosis. Both seem to be affected by multiple pathways and related polymorphisms. In our study the AC variant of MTHFR A1298C is neither associated with response nor with improved outcome, but with poor prognosis in the subgroup of neoadjuvantly treated patients. However there exists data in literature, which correspond to our findings on the MTHFR A1298C 
polymorphism. Locally advanced adenocarcinoma of the stomach treated with cisplatin and 5-FU based chemotherapy with the variants of MTHFR A1298C (AC, CC) were associated with higher risks of recurrence and death in gastric cancer patients in a recent paper from our group [23] including 244 identical patients. Another study including unresectable, advanced gastric carcinomas reported similar results with shorter survival times of A1298C variants (AC and CC) (6.6 months median survival versus 18.5 months of the wild type $(\mathrm{AA}), \mathrm{p}=0.001$ ) [48]. A pathway driven approach including amongst others both MTHFR polymorphisms showed conflicting results for esophageal cancer compared to ours. Longer survival and recurrence-free survival times could be shown for the MTHFR A1298C variants types $(p=0.01)$ as well as for combination of variant alleles at both loci $(\mathrm{AC}+\mathrm{CC})$ compared to the individuals with one wild type allele (AA) [49]. Two other studies reported a survival benefit for the TT variant of C677T $[28,50]$ in patients with fluorouracil-based chemotherapy. In the adjuvant setting the CC genotype of MTHFR C677T was associated with shorter recurrence-free survival $(\mathrm{p}=0.031)$ [28]. However, several other studies could not show any prognostic impact of one the MTHFR polymorphisms for patients with esophagogastric cancer [22,51-53], so that results remain controversial (see Table 7).

As in our previous published studies [16,22,23] we could not show an association between the two polymorphisms and response to neoadjuvant treatment.
Most of the studies on MTHFR polymorphisms and upper gastrointestinal cancer could not reproduce the association of response with fluoropyrimidine based chemotherapy having been described for other cancer types, mainly advanced colon carcinoma [45,56,57], apart from one Chinese study for the TT genotype of the MTHFR C677T polymorphism and response [54].

In one study including metastatic gastroesophageal adenocarcinomas, the AC variant of MTHFR A1298C was associated with lower response rates (27\% responder in contrast to 48 and $46 \%$ responder for AA and CC genotype, $\mathrm{p}=0.053$ ) [52]. Other studies could not confirm the effect of MTHFR C677T and A1298C variants in response to chemotherapy [22,23,51] (see Table 7).

The conflicting and heterogenous findings highlight the demand on comprehensive pathway-based approaches for the prediction of response and prognosis by genetic polymorphisms as a potentially more successful and promising strategy. Beside the activity of the MTHFR, also the individual folate intake might influence outcome. Individuals with a high dietary folate intake are described to have a lower risk of developing gastrointestinal cancer. Consequently not only MTHFR polymorphisms, which play an important role in folate metabolism but also folate intake could be associated with prognosis in patients with esophagogastric carcinoma [50].

Further general problems of the presented studies are their heterogeneity and lack of comparability with respect to the number of patients included, inclusion

Table 7 MTHFR polymorphisms in literature

\begin{tabular}{|c|c|c|c|c|c|c|c|c|c|}
\hline Author & Year & Polymorphism & $\mathbf{n}$ & Treatment & Tumor entity & Response (p-value) & Prognosis ( $p$-value) & Genotypes & In P F \\
\hline \multirow[t]{2}{*}{ Ott [22] } & 2006 & MTHFR C677T & 235 & neo CTx 135 & AEGII/II/GC & 0.14 & 0.14 & & \\
\hline & & & & OP 103 & AEGII/II/GC & - & 0.23 & & \\
\hline \multirow[t]{4}{*}{ Ott [23] } & 2011 & MTHFR C677T & 258 & neo CTx & AEGI 114 & n.s. & n.s. & & \\
\hline & & & & & AEGII-GC 144 & n.s. & n.s. & & \\
\hline & & MTHFR A1298C & 258 & neo CTx & AEGI 114 & n.s. & n.s. & & \\
\hline & & & & & AEGII-GC 144 & n.s. & 0.02 & $A A>C C>A C$ & yes \\
\hline Huang [28] & 2008 & MTHFR C677T & 116 & $\operatorname{adj} C T x$ & GC & & 0.04 & $\Pi / C T>C C$ & 0.056 \\
\hline \multirow[t]{2}{*}{ Wu [49] } & 2006 & MTHFR C677T & 210 & neoCTx/RCTx & AEGI 174/SCC 36 & n.a. & n.s. & & \\
\hline & & MTHFR A1298C & 210 & neoCTx/RCTx & AEGI 174/SCC36 & n.a. & 0.011 & $\mathrm{AC} / \mathrm{CC}>\mathrm{AA}$ & n.a. \\
\hline Ruzzo [51] & 2006 & MTHFR C677T & 175 & pall CTx & GC & 0.2 & n.s. & & \\
\hline Chen [48] & 2010 & MTHFR A1298C & $16 / 73$ & pall $C T x$ & GC & - & $<0.001$ & $\mathrm{AA}>\mathrm{AC} / \mathrm{CC}$ & n.a. \\
\hline Lu [54] & 2004 & MTHFR C677T & 75 & pall $C T x$ & GC & 0.001 & - & $\Pi \mathrm{T}>\mathrm{CC} / \mathrm{CT}$ & $<0.001$ \\
\hline \multirow[t]{2}{*}{ Goekkurt [52] } & 2009 & MTHFR C677T & 134 & pall CTx & AEG/GC & 0.214 & 0.319 & & \\
\hline & & MTHFR A1298C & 134 & pall CTx & AEG/GC & 0.053 & 0.524 & & \\
\hline Goekkurt [55] & 2006 & MTHFR C677T & 52 & pall CTx & GC & 0.099 & n.s. & & \\
\hline Shitara [50] & 2010 & MTHFR C677T & 132 & pall CTx & GC & n.a. & 0.039 & $\Pi>\mathrm{CC} / \mathrm{CT}$ & \\
\hline Lee [53] & 2005 & MTHFR C677T & 40 & $\operatorname{adj} C T x$ & GC & & 0.90 & & \\
\hline
\end{tabular}

neo = neoadjuvant, $C T x=$ chemotherapy, $\mathrm{RCTx}=$ radiochemotherapy, $\mathrm{OP}=$ primarily resected, adj $=$ adjuvant, pall $=$ palliative, $\mathrm{AEG}=$ adenocarcinoma of the esophagus/esophagusgastric junction, $\mathrm{GC}=$ gastric cancer, n.s. = not significant, n.a. = not applied, In P F = independent prognostic factor. 
criteria (type of tumor, tumor stage), different treatment concepts (palliative treatment versus curative treatment including radical surgery) and different genotypes. The change from UICC $6^{\text {th }}$ to $7^{\text {th }}$ might especially complicate the comparability of old and recent studies, as AEG II and III are classified differently [58]. To exclude this bias we repeated the analyses for both classifications and could show that in patients with AEG I or adenocarcinomas of the esophagogastric junction, the MTHFR polymorphisms did not have prognostic impact related to our recent paper [23].

Our study has some limitations. The first limitation is the inclusion of patients, on which we already reported recently, however, the follow-up was significantly extended [22,23]. Secondly it is a retrospective exploratory study and therefore has the typical disadvantages of this study type.

But in contrast to many other studies, we analyzed patients being primarily resected without perioperative concepts to evaluate the prognostic impact of the described polymorphisms apart from response to chemotherapy as a simple prognostic factor. In our study the prognostic impact of MTHFR A1298C could not be demonstrated in primarily resected patients. This leads to the conclusion that response to treatment plays an important role for influence on survival of the different genotypes, despite the effect on response could not be measured by a correlation with clinical and histopathological response in this study. A further strength of our study is the relatively high number of patients, to our knowledge the largest published series, the inclusion of adenocarcinomas only and a homogenous 5-FU containing preoperative treatment followed by resection.

\section{Conclusions}

The AC genotype of the MTHFR A1298C was associated with a poor prognosis in neoadjuvantly treated gastric cancer patients, although there was no association with clinically or histopathologically assessed response to chemotherapy. This gives a clear hint towards the modulation of prognosis by chemotherapy, which cannot be measured by the available methods of response evaluation. Large patient numbers and pathway driven approaches seem necessary to evaluate the prognostic impact of polymorphisms in patients with esophagogastric adenocarcinomas to tailor treatment in the future.

\section{Abbreviations}

MTHFR: Methylentetrahydrofolate reductase; AEG: Carcinoma of the esophagogastric junction; SNP: Single nucleotide polymorphism; GC: Gastric cancer; PCR: Polymerase chain reaction; TRG: Tumor regression grade.

\section{Competing interests}

The authors declare that they have no competing interests.

\section{Authors' contributions}

SB participated in acquisition of data, statistical analysis, interpretation of data and manuscript writing and final approval of the manuscript; SK carried out the genotyping, participated in study design, interpretation of data and manuscript writing and final approval of the manuscript, GK participated in study design, interpretation of data and critical revision of the manuscript and final approval of the manuscript WW participated in interpretation of data, critical revision of the manuscript and final approval of the manuscript, FL participated in study design, interpretation of data, critical revision of the manuscript and final approval of the manuscript, RL participated in study design, interpretation of data, critical revision of the manuscript and final approval of the manuscript, CS participated in interpretation of data, critical revision of the manuscript and final approval of the manuscript, TB participated in statistical analysis, critical revision of the manuscript and final approval of the manuscript, KB participated in study design, interpretation of data, critical revision of the manuscript and final approval of the manuscript, RK carried out the genotyping, participated in study design, interpretation of data, critical revision of the manuscript and final approval of the manuscript, $\mathrm{KO}$ participated in study design, acquisition of data, interpretation of data, manuscript writing, critical revision of the manuscript and final approval of the manuscript. All authors have read and given final approval of the version to be published.

\section{Acknowledgement}

Presented in part at the International Gastric Cancer Congress 2013 in Verona and awarded by a poster award. We thank Catherine Bernaciak PhD for revising the manuscript.

\section{Author details}

${ }^{1}$ Department of Surgery, University Hospital of Heidelberg, Im Neuenheimer Feld 110, Heidelberg 69120, Germany. ' DKFZ, University of Heidelberg, Heidelberg, Germany. ${ }^{3}$ Institute of pathology, Technische Universitaet, Muenchen, Munich, Germany. ${ }^{4}$ Institute of pathology, University of Heidelberg, Heidelberg, Germany. ${ }^{5}$ University Cancer Center Leipzig (UCCL), Leipzig, Germany. ${ }^{6}$ Institute of pathology, University of Bern, Bern, Switzerland. ${ }^{7}$ National Center of Tumor Diseases, University of Heidelberg, Heidelberg, Germany. ${ }^{8}$ Medical Biometry, University of Heidelberg, Heidelberg, Germany.

Received: 8 July 2013 Accepted: 26 November 2013 Published: 3 February 2014

\section{References}

1. Cunningham D, Allum W, Stenning S, Thompson J, Van de Velde C, Nicolson M, Scarffe J, Lofts F, Falk S, Iveson T, Smith D, Langley R, Verma M, Weeden S, Chua Y: MAGIC trial participants: perioperative chemotherapy versus surgery alone for resectable gastroesophageal cancer. N Engl J Med 2006, 355(1):11-20.

2. Ychou $M$, Boige $V$, Pignon JP, Conroy $T$, Bouché $O$, Lebreton $G$, Ducourtieux M, Bedenne L, Fabre JM, Saint-Aubert B, Genève J, Lasser P, Rougier P: Perioperative chemotherapy compared with surgery alone for resectable gastroesophageal adenocarcinoma: an FNCLCC and FFCD multicenter phase III trial. J Clin Oncol 2011, 29(13):1715-1721.

3. van Hagen $P$, Hulshof MC, van Lanschot JJ, Steyerberg EW, van Berge Henegouwen MI, Wijnhoven BP, Richel DJ, Nieuwenhuijzen GA, Hospers GA, Bonenkamp JJ, Cuesta MA, Blaisse RJ, Busch OR, ten Kate FJ, Creemers GJ, Punt CJ, Plukker JT, Verheul HM, Spillenaar Bilgen EJ, van Dekken H, van der Sangen MJ, Rozema T, Biermann K, Beukema JC, Piet AH, van Rij CM, Reinders JG, Tilanus HW, van der Gaast A, Group C: Preoperative chemoradiotherapy for esophageal or junctional cancer. N Engl J Med 2012, 366(22):2074-2084.

4. Sjoquist KM, Burmeister BH, Smithers BM, Zalcberg JR, Simes RJ, Barbour A, Gebski V, Group AG-IT: Survival after neoadjuvant chemotherapy or chemoradiotherapy for resectable oesophageal carcinoma: an updated meta-analysis. Lancet Oncol 2011, 12(7):681-692.

5. Becker K, Langer R, Reim D, Novotny A, Meyer zum Buschenfelde C, Engel J, Friess $\mathrm{H}$, Hofler $\mathrm{H}$ : Significance of histopathological tumor regression after neoadjuvant chemotherapy in gastric adenocarcinomas: a summary of 480 cases. Ann Surg 2011, 253(5):934-939. 
6. Lowy A, Mansfield P, Leach S, Pazdur R, Dumas P, Ajani J: Response to neoadjuvant chemotherapy best predicts survival after curative resection of gastric cancer. Ann Surg 1999, 229(3):303-308

7. Meredith KL, Weber JM, Turaga KK, Siegel EM, McLoughlin J, Hoffe S, Marcovalerio M, Shah N, Kelley S, Karl R: Pathologic response after neoadjuvant therapy is the major determinant of survival in patients with esophageal cancer. Ann Surg Oncol 2010, 17(4):1159-1167.

8. Vallböhmer D, Hölscher AH, DeMeester S, DeMeester T, Salo J, Peters J, Lerut T, Swisher SG, Schröder W, Bollschweiler E, Hofstetter W: A multicenter study of survival after neoadjuvant radiotherapy/chemotherapy and esophagectomy for ypTONOMORO esophageal cancer. Ann Surg 2010, 252(5):744-749.

9. Blank S, Bläker H, Schaible A, Lordick F, Grenacher L, Buechler M, Ott K: Impact of pretherapeutic routine clinical staging for the individualization of treatment in gastric cancer patients. Langenbecks Arch Surg 2012, 397(1):45-55.

10. Park SR, Kim MJ, Ryu KW, Lee JH, Lee JS, Nam BH: Prognostic value of preoperative clinical staging assessed by computed tomography in resectable gastric cancer patients: a viewpoint in the era of preoperative treatment. Ann Surg 2010, 251(3):428-345

11. Piessen G, Messager M, Leteurtre E, Jean-Pierre T, Mariette C: Signet ring cell histology is an independent predictor of poor prognosis in gastric adenocarcinoma regardless of tumoral clinical presentation. Ann Surg 2009, 250(6):878-887.

12. Inoue $K$, Nakane $Y$, Michiura T, Nakai K, liyama H, Sato M, Okumura S, Yamamichi K, Hioki K: Histopathological grading does not affect survival after R0 surgery for gastric cancer. Eur J Surg Oncol 2002, 28(6):633-636.

13. Hochwald SN, Kim S, Klimstra DS, Brennan MF, Karpeh MS: Analysis of 154 actual five-year survivors of gastric cancer. J Gastrointest Surg 2000, 4(5):520-525.

14. Schmidt SC, Schlechtweg N, Veltzke-Schlieker W, Thuss-Patience P, Pratschke J, Neuhaus P, Schumacher G: Clinical and pathological prognostic factors for cancers of the esophagogastric junction. Zentralb/ Chir 2009, 134(5):455-461.

15. Bang YJ, Van Cutsem E, Feyereislova A, Chung HC, Shen L, Sawaki A, Lordick F, Ohtsu A, Omuro Y, Satoh T, Aprile G, Kulikov E, Hill J, Lehle M, Rüschoff J, Kang YK, Investigators TT: Trastuzumab in combination with chemotherapy versus chemotherapy alone for treatment of HER2positive advanced gastric or gastro-oesophageal junction cancer (ToGA): a phase 3, open-label, randomised controlled trial. Lancet 2010, 376(9742):687-697.

16. Ott K, Lordick F, Becker K, Ulm K, Siewert J, Höfler H, Keller G: GlutathioneS-transferase $\mathrm{P} 1, \mathrm{~T} 1$ and $\mathrm{M} 1$ genetic polymorphisms in neoadjuvanttreated locally advanced gastric cancer: GSTM1-present genotype is associated with better prognosis in completely resected patients. Int J Colorectal Dis 2008, 23(8):773-782.

17. Ott K, Vogelsang H, Mueller J, Becker K, Müller M, Fink U, Siewert JR, Höfler $\mathrm{H}$, Keller G: Chromosomal instability rather than p53 mutation is associated with response to neoadjuvant cisplatin-based chemotherapy in gastric carcinoma. Clin Cancer Res 2003, 9(6):2307-2315

18. Bachmann K, Shahmiri S, Kaifi J, Schurr P, Mann O, Rawnaq T, Block S, Kalinin V, Izbicki JR, Strate T: Polymorphism Arg290Arg in esophagealcancer-related gene 1 (ECRG1) is a prognostic factor for survival in esophageal cancer. J Gastrointest Surg 2009, 13(2):181-187.

19. Bradbury PA, Zhai R, Hopkins J, Kulke MH, Heist RS, Singh S, Zhou W, Ma C, Xu W, Asomaning K, Ter-Minassian M, Wang Z, Su L, Christiani DC, Liu G: Matrix metalloproteinase 1, 3 and 12 polymorphisms and esophageal adenocarcinoma risk and prognosis. Carcinogenesis 2009, 30(5):793-798.

20. Bradbury PA, Zhai R, Ma C, Xu W, Hopkins J, Kulke MJ, Asomaning K, Wang Z, Su L, Heist RS, Lynch TJ, Wain JC, Christiani D, Liu G: Vascular endothelial growth factor polymorphisms and esophageal cancer prognosis. Clin Cancer Res 2009, 15(14):4680-4685.

21. Cescon DW, Bradbury PA, Asomaning K, Hopkins J, Zhai R, Zhou W, Wang Z, Kulke M, Su L, Ma C, Xu W, Marshall AL, Heist RS, Wain JC, Lynch TJ, Christiani DC, Liu G: p53 Arg72Pro and MDM2 T309G polymorphisms, histology, and esophageal cancer prognosis. Clin Cancer Res 2009, 15(9):3103-3109.

22. Ott K, Vogelsang $H$, Marton N, Becker $K$, Lordick F, Kobl M, Schuhmacher C, Novotny A, Mueller J, Fink U, Ulm K, Siewert JR, Höfler H, Keller G: The thymidylate synthase tandem repeat promoter polymorphism: a predictor for tumorrelated survival in neoadjuvant treated locally advanced gastric cancer. Int J Cancer 2006, 119(12):2885-2894
23. Ott K, Rachakonda PS, Panzram B, Keller G, Lordick F, Becker K, Langer R, Buechler M, Hemminki K, Kumar R: DNA repair gene and MTHFR gene polymorphisms as prognostic markers in locally advanced adenocarcinoma of the esophagus or stomach treated with cisplatin and 5-fluorouracilbased neoadjuvant chemotherapy. Ann Surg Oncol 2011, 18(9):2688-2698.

24. Marcuello E, Altés A, Menoyo A, Rio ED, Baiget M: Methylenetetrahydrofolate reductase gene polymorphisms: genomic predictors of clinical response to fluoropyrimidine-based chemotherapy? Cancer Chemother Pharmacol 2006, 57(6):835-840.

25. Sohn KJ, Croxford R, Yates Z, Lucock M, Kim Yl: Effect of the methylenetetrahydrofolate reductase C677T polymorphism on chemosensitivity of colon and breast cancer cells to 5-fluorouracil and methotrexate. J Natl Cancer Inst 2004, 96(2):134-144.

26. Toffoli G, De Mattia E: Pharmacogenetic relevance of MTHFR polymorphisms. Pharmacogenomics 2008, 9(9):1195-1206.

27. Ueland PM, Hustad S, Schneede J, Refsum H, Vollset SE: Biological and clinical implications of the MTHFR C677T polymorphism. Trends Pharmacol Sci 2001, 22(4):195-201.

28. Huang ZH, Hua D, Li LH: The polymorphisms of TS and MTHFR predict survival of gastric cancer patients treated with fluorouracil-based adjuvant chemotherapy in Chinese population. Cancer Chemother Pharmacol 2009 63(5):911-918

29. Wang Z, Chen JQ, Liu JL, Qin XG, Huang Y: Polymorphisms in ERCC1, GSTs, TS and MTHFR predict clinical outcomes of gastric cancer patients treated with platinum/5-Fu-based chemotherapy: a systematic review. BMC Gastroenterol 2012, 12:137.

30. Sarbia M, Stahl M, von Weyhern C, Weirich G, Pühringer-Oppermann F: The prognostic significance of genetic polymorphisms (Methylenetetrahydrofolate Reductase C677T, Methionine Synthase A2756G, Thymidilate Synthase tandem repeat polymorphism) in multimodally treated oesophageal squamous cell carcinoma. $\mathrm{Br} J$ Cancer 2006, 94(2):203-207.

31. Weisberg I, Tran P, Christensen B, Sibani S, Rozen R: A second genetic polymorphism in methylenetetrahydrofolate reductase (MTHFR) associated with decreased enzyme activity. Mol Genet Metab 1998, 64(3):169-172.

32. Friedman G, Goldschmidt N, Friedlander Y, Ben-Yehuda A, Selhub J, Babaey S, Mendel M, Kidron M, Bar-On H: A common mutation A1298C in human methylenetetrahydrofolate reductase gene: association with plasma total homocysteine and folate concentrations. J Nutr 1999, 129(9):1656-1661.

33. Ott K, Sendler A, Becker K, Dittler H, Helmberger H, Busch R, Kollmannsberger C, Siewert J, Fink U: Neoadjuvant chemotherapy with cisplatin, 5-FU, and leucovorin (PLF) in locally advanced gastric cancer: a prospective phase II study. Gastric Cancer 2003, 6(3):159-167.

34. Ott K, Bader F, Lordick F, Feith M, Bartels H, Siewert J: Surgical factors influence the outcome after Ivor-Lewis esophagectomy with intrathoracic anastomosis for adenocarcinoma of the esophagogastric junction: a consecutive series of 240 patients at an experienced center. Ann Surg Oncol 2009, 16(4):1017-1025.

35. Uzunoglu FG, Reeh M, Kutup A, Izbicki JR: Surgery of esophageal cancer. Langenbecks Arch Surg 2013, 398(2):189-193

36. Hulscher JB, van Sandick JW, de Boer AG, Wijnhoven BP, Tijssen JG, Fockens P, Stalmeier PF, ten Kate FJ, van Dekken H, Obertop H, Tilanus HW van Lanschot JJ: Extended transthoracic resection compared with limited transhiatal resection for adenocarcinoma of the esophagus. N Engl J Med 2002, 347(21):1662-1669.

37. Bozzetti F, Marubini E, Bonfanti G, Miceli R, Piano C, Gennari L: Subtotal versus total gastrectomy for gastric cancer: five-year survival rates in a multicenter randomized italian trial. Italian gastrointestinal tumor study group. Ann Surg 1999, 230(2):170-178.

38. Ott K, Fink U, Becker K, Stahl A, Dittler H, Busch R, Stein H, Lordick F, Link T, Schwaiger M, Siewert J, Weber W: Prediction of response to preoperative chemotherapy in gastric carcinoma by metabolic imaging: results of a prospective trial. J Clin Oncol 2003, 21(24):4604-4610.

39. Weber WA, Ott K, Becker K, Dittler HJ, Helmberger H, Avril NE, Meisetschläger G, Busch R, Siewert JR, Schwaiger M, Fink U: Prediction of response to preoperative chemotherapy in adenocarcinomas of the esophagogastric junction by metabolic imaging. J Clin Oncol 2001, 19(12):3058-3065.

40. Fink U, Schuhmacher C, Stein HJ, Busch R, Feussner H, Dittler HJ, Helmberger A, Böttcher K, Siewert JR: Preoperative chemotherapy for stage III-IV gastric 
carcinoma: feasibility, response and outcome after complete resection. Br J Surg 1995, 82(9):1248-1252.

41. Becker K, Mueller J, Schulmacher C, Ott K, Fink U, Busch R, Böttcher K, Siewert J, Höfler $\mathrm{H}$ : Histomorphology and grading of regression in gastric carcinoma treated with neoadjuvant chemotherapy. Cancer 2003, 98(7):1521-1530.

42. Brattström L, Wilcken DE, Ohrvik J, Brudin L: Common methylenetetrahydrofolate reductase gene mutation leads to hyperhomocysteinemia but not to vascular disease: the result of a meta-analysis. Circulation 1998, 98(23):2520-2526.

43. Böttiger AK, Hurtig-Wennlöf A, Sjöström M, Yngve A, Nilsson TK: Association of total plasma homocysteine with methylenetetrahydrofolate reductase genotypes $677 \mathrm{C}>\mathrm{T}, 1298 \mathrm{~A}>\mathrm{C}$, and 1793G $>\mathrm{A}$ and the corresponding haplotypes in Swedish children and adolescents. Int J Mol Med 2007, 19(4):659-665.

44. Lievers KJ, Boers GH, Verhoef $P$, den Heijer M, Kluijtmans LA, van der Put NM, Trijbels FJ, Blom HJ: A second common variant in the methylenetetrahydrofolate reductase (MTHFR) gene and its relationship to MTHFR enzyme activity, homocysteine, and cardiovascular disease risk. J Mol Med (Berl) 2001, 79(9):522-528

45. Etienne MC, Formento $J$, Chazal M, Francoual M, Magné N, Formento $P$, Bourgeon A, Seitz JF, Delpero JR, Letoublon C, Pezet D, Milano G: Methylenetetrahydrofolate reductase gene polymorphisms and response to fluorouracil-based treatment in advanced colorectal cancer patients. Pharmacogenetics 2004, 14(12):785-792.

46. Etienne MC, Ilc K, Formento JL, Laurent-Puig P, Formento P, Cheradame S, Fischel $J$, Milano G: Thymidylate synthase and methylenetetrahydrofolate reductase gene polymorphisms: relationships with 5-fluorouracil sensitivity. Br J Cancer 2004, 90(2):526-534.

47. De Mattia E, Toffoli G: C677T and A1298C MTHFR polymorphisms, a challenge for antifolate and fluoropyrimidine-based therapy personalisation. Eur J Cancer 2009, 45(8):1333-1351.

48. Chen JS, Chao Y, Bang YJ, Roca E, Chung HC, Palazzo F, Kim YH, Myrand SP, Mullaney BP, Shen LJ, Linn C: A phase I/II and pharmacogenomic study of pemetrexed and cisplatin in patients with unresectable, advanced gastric carcinoma. Anticancer Drugs 2010, 21(8):777-784.

49. Wu X, Gu J, Wu TT, Swisher SG, Liao Z, Correa AM, Liu J, Etzel CJ, Amos Cl, Huang M, Chiang SS, Milas L, Hittelman WN, Ajani JA: Genetic variations in radiation and chemotherapy drug action pathways predict clinical outcomes in esophageal cancer. J Clin Oncol 2006, 24(23):3789-3798.

50. Shitara K, Muro K, Ito S, Sawaki A, Tajika M, Kawai H, Yokota T, Takahari D, Shibata T, Ura T, Ito H, Hosono S, Kawase T, Watanabe M, Tajima K, Yatabe Y, Tanaka H, Matsuo K: Folate intake along with genetic polymorphisms in methylenetetrahydrofolate reductase and thymidylate synthase in patients with advanced gastric cancer. Cancer Epidemiol Biomarkers Prev 2010, 19(5):1311-1319.

51. Ruzzo A, Graziano F, Kawakami K, Watanabe G, Santini D, Catalano V, Bisonni R, Canestrari E, Ficarelli R, Menichetti ET, Mari D, Testa E, Silva R, Vincenzi B, Giordani P, Cascinu S, Giustini L, Tonini G, Magnani M: Pharmacogenetic profiling and clinical outcome of patients with advanced gastric cancer treated with palliative chemotherapy. J Clin Oncol 2006, 24(12):1883-1891.

52. Goekkurt E, Al-Batran SE, Hartmann JT, Mogck U, Schuch G, Kramer M, Jaeger E, Bokemeyer C, Ehninger G, Stoehlmacher J: Pharmacogenetic analyses of a phase III trial in metastatic gastroesophageal adenocarcinoma with fluorouracil and leucovorin plus either oxaliplatin or cisplatin: a study of the arbeitsgemeinschaft internistische onkologie. J Clin Oncol 2009, 27(17):2863-2873.

53. Lee J, Jeong CK, Hong SP, Chong SY, Oh D, Hwang SG, Ahn DH, Kim S, Han $\mathrm{JH}, \mathrm{Kim}$ NK: Clinical significance of thymidylate synthase and methylenetetrahydrofolate reductase gene polymorphism in Korean patients with gastric cancer. Korean J Gastroenterol 2005, 46(1):32-38.

54. Lu JW, Gao CM, Wu JZ, Sun XF, Wang L, Feng JF: Relationship of methylenetetrahydrofolate reductase C677T polymorphism and chemosensitivity to 5-fluorouracil in gastric carcinoma. Ai Zheng 2004, 23(8):958-962.

55. Goekkurt E, Hoehn S, Wolschke C, Wittmer C, Stueber C, Hossfeld DK, Stoehlmacher J: Polymorphisms of glutathione S-transferases (GST) and thymidylate synthase (TS)-novel predictors for response and survival in gastric cancer patients. Br J Cancer 2006, 94(2):281-286.

56. Cohen V, Panet-Raymond V, Sabbaghian N, Morin I, Batist G, Rozen R: Methylenetetrahydrofolate reductase polymorphism in advanced colorectal cancer: a novel genomic predictor of clinical response to fluoropyrimidine-based chemotherapy. Clin Cancer Res 2003, 9(5):1611-1615.

57. Jakobsen A, Nielsen JN, Gyldenkerne N, Lindeberg J: Thymidylate synthase and methylenetetrahydrofolate reductase gene polymorphism in normal tissue as predictors of fluorouracil sensitivity. J Clin Oncol 2005, 23(7):1365-1369.

58. Sobin LH, Compton CC: TNM seventh edition: what's new, what's changed: communication from the International Union Against Cancer and the American Joint Committee on Cancer. Cancer 2010, 116(22):5336-5339.

doi:10.1186/1471-2407-14-58

Cite this article as: Blank et al:: A retrospective comparative exploratory study on two Methylentetrahydrofolate Reductase (MTHFR) polymorphisms in esophagogastric cancer: the A1298C MTHFR polymorphism is an independent prognostic factor only in neoadjuvantly treated gastric cancer patients. BMC Cancer 2014 14:58.

\section{Submit your next manuscript to BioMed Central and take full advantage of:}

- Convenient online submission

- Thorough peer review

- No space constraints or color figure charges

- Immediate publication on acceptance

- Inclusion in PubMed, CAS, Scopus and Google Scholar

- Research which is freely available for redistribution

Submit your manuscript at www.biomedcentral.com/submit
C Biomed Central 